\title{
Raconter des histoires est une chose sérieuse : L'Afrique fantôme de Michel Leiris
}

\author{
Alberto M. Sobrero \\ Traducteur : Silvia Disegni
}

\section{OpenEdition \\ Journals}

Édition électronique

URL : http://journals.openedition.org/recherchestravaux/581

DOI : 10.4000/recherchestravaux.581

ISSN : 1969-6434

Éditeur

UGA Éditions/Université Grenoble Alpes

Édition imprimée

Date de publication : 15 mai 2013

Pagination : 53-67

ISBN : 978-2-84310-248-6

ISSN : 0151-1874

Référence électronique

Alberto M. Sobrero, «Raconter des histoires est une chose sérieuse : L'Afrique fantôme de Michel Leiris », Recherches \& Travaux [En ligne], 82 | 2013, mis en ligne le 15 novembre 2014, consulté le 08 septembre 2020. URL : http://journals.openedition.org/recherchestravaux/581 ; DOI : https://doi.org/ 10.4000/recherchestravaux.581 
Alberto M. Sobrero

Université La Sapienza, Rome

\title{
Raconter des histoires est une chose sérieuse: L'Afrique fantôme de Michel Leiris
}

\author{
(Traduit de l'italien par Silvia Disegni)
}

Raconter des histoires est une chose sérieuse; dès notre naissance et jusqu'à notre mort, nous ne faisons que raconter, nous sommes obsédés par le besoin de chercher du regard, avec les mots, le sens de ce qui arrive en nous et hors de nous, par le besoin de reconnaître le monde. Nous ne pouvons faire autrement. Les philosophes ont défini une telle disposition de la manière la plus neutre qui soit : in alium tendere, intentionnalité. Ils n'ont pas parlé de pensée, parce que l'acte de penser implique la volonté, alors que nous racontons le monde même sans le vouloir et encore, parfois, ce sont les histoires qui nous assaillent, nous poursuivent et s'imposent à notre esprit, indépendamment de notre volonté et contre elle. Ils n'ont pas parlé non plus de compréhension, car il nous semble souvent que les mots et les sentiments ne suffisent pas à faire comprendre ce qui nous arrive : la narration devient impossible, elle cède la place à l'angoisse, à la gorge qui se serre. Ils parlent encore moins d'explication, qui prétendrait démontrer la vérité du sens que nous donnons au monde extérieur.

En ce sens, on peut bien dire que la philosophie a anticipé la science. Aujourd'hui, les évolutionnistes - et en particulier la branche cognitiviste de l'évolutionnisme (j'utilise le terme "cognitiviste» dans l'acception de Jérôme Bruner ${ }^{1}$ - nous expliquent que la capacité d'imaginer des histoires, de raconter, ou, plus simplement, la capacité de donner du sens et de construire des contextes, est un mécanisme fondateur de l'histoire de la vie et qu'il a sans doute offert à l'évolution mentale des avantages croissants

I. J. Bruner, Acts of Meaning, Cambridge, Harvard University Press, 1990. 
et rapides, analogues à ceux qu'a pu produire dans l'histoire de l'évolution du corps la posture verticale ${ }^{2}$, grâce à laquelle nous avons libéré nos mains. Dans l'évolution mentale, nous nous sommes progressivement libérés de nos instincts, des histoires déjà écrites dans notre esprit, et nous avons commencé à expérimenter la possibilité de raconter de nouvelles histoires. Les animaux aussi racontent le monde à leur manière (et les éthologues ont plus ou moins compris comment), à une différence près : leurs histoires, qu'on les appelle modules ou instincts, sont déjà en grande partie racontées et bien imprimées dans leur esprit/cerveau.

Marcher à quatre pattes puis sur deux pieds, passer de l'instinct au besoin d'imaginer des histoires ont dû être des changements particulièrement éprouvants, mais ils ont permis un progrès. Plus on savait raconter, plus on savait donner du sens aux choses, par-delà la véridicité des histoires, plus augmentait la possibilité de survie de l'espèce, sa propre fitness. Pendant quelques millions d'années, l'histoire de l'hominisation a été celle de la capacité du genre Homo et de l'espèce sapiens de passer des observations et des idées les plus générales et les plus grossières à des histoires toujours plus différenciées et particulières. Et, en des temps pas aussi éloignés qu'on le croit, l'affinement des compétences linguistiques a joué un rôle déterminant dans ce processus. Le monde, quel qu'il soit, est toujours le même, mais nous l'avons raconté différemment, nous y avons trouvé de plus en plus de détails, en élaborant des observations, des sensations et des sentiments différents. Nous l'avons rempli d'intentionnalité (à savoir, à la fois imagination et compréhension...).

Néanmoins, même en voulant rester bref, les choses ne sont pas si simples. Ce que nous avons été physiquement a laissé bien des traces dans notre corps. A posteriori, nous pouvons être assez satisfaits du travail de la sélection naturelle, mais quelques erreurs ont quand même été commises. Il y a eu quelque rapidité. Des étapes ont été franchies trop rapidement, que nous payons par des maux de dents, des problèmes de dos et probablement par un certain nombre de maladies génétiques. Ainsi en est-il également de notre cerveau : de temps en temps affleurent des histoires anciennes, des traces lointaines de l'instinct. Mais ce n'est pas le problème le plus grave. Ce sont là des questions auxquelles pourrait penser la culture. Le fait est que la prétention de raconter le monde présente un défaut de constitution évident, un point aveugle qui n'est pas, en l'occurrence, le point aveugle de la rétine (une telle limite découle précisément d'une mise au point imparfaite de la sélection naturelle). Ce n'est pas un défaut des sens, mais plutôt un défaut

2. G. M. Edelman, Bright Air, Brillant Fire. On the Matter of the Mind, New York, Basic Books Inc., I992; A. R. Damasio, The Feeling of What Happens, Londres, Vintage, 2000. 
de l'esprit : c'est le point aveugle qui est en jeu quand nous prétendons nous raconter nous-mêmes. Nous ne pouvons sortir de nous-mêmes sans tomber dans le célèbre paradoxe consistant à vouloir sortir du marécage en se tirant soi-même par les cheveux. Et les choses se compliquent quand nous voulons raconter la vie des autres. Pour reprendre les paroles de l'Evariste Carriego de Borges :

Qu'un individu veuille réveiller dans un autre individu des souvenirs qui n'appartiennent qu'à une tierce personne, c'est un paradoxe évident. Réaliser ce paradoxe en toute tranquillité, tel est le vouloir innocent de tout biographe.

Le rapprochement entre la limite physique de l'œil et celui de l'esprit est bien posé, parce que pour donner de la lumière au point aveugle de la rétine, le cerveau fait ce que nous faisons quand nous savons nous raconter. Dans les deux cas, nous contournons le paradoxe, en faisant semblant de rien : le cerveau le contourne en utilisant la lumière et les images du contexte; "en reconstruisant de mémoire", nous faisons semblant de croire que le sujet qui raconte est différent de celui que nous racontons, nous faisons semblant de sortir de nous-mêmes, de nous observer du dehors, de raconter et de nous raconter toujours les mêmes histoires. Le fait est pourtant qu'à travers ce point aveugle, à travers les histoires habituelles, peut s'insinuer une crainte plus profonde : la sensation de la vacuité du «je» raconté, et avec elle, du non-sens de notre vie.

Michel Leiris, dont nous parlerons ici, s'est trouvé au bord de ce néant, vers lequel il s'est senti attiré presque au terme de son parcours africain. Il ne s'en est sorti que pour des "questions de peau, de civilisation, de langue».

Nous comprendrons vite la raison de ces prémisses. Mais venons-en maintenant au rapport littérature/anthropologie. La question est vaste. Essayons de passer brièvement en revue les domaines d'intérêt possibles. Sur le plan théorique, on va des études sur le rapport entre littérature populaire et sens commun aux grandes constructions théoriques de Propp, Lévi-Strauss, Freud, Jung, Frye, Bakhtine, Gadamer ou Ricœur; de l'utilisation par certains anthropologues des textes littéraires, dont ils se servent comme de sources ethnographiques, à l'usage que font les critiques littéraires des méthodes de l'anthropologie, considérées comme des dispositifs possibles d'analyse textuelle. Sur le plan performatif, surtout au cours de ces vingt ou trente dernières années, l'attention s'est focalisée sur les anthropologues (plus ou moins accomplis, plus ou moins manqués) qui écrivent des œuvres littéraires, journaux intimes, autobiographies, romans (tout au long de l'histoire de l'anthropologie et jusqu'à Kurt Vonnegut, à Amitav Ghosh, Saul Bellow ou, en Italie, Giulio Angioni), et d'un autre côté, sur les écrivains (inutile de les mentionner) qui ont travaillé 
et travaillent sur des scénarios, des thématiques, des métaphores à caractère anthropologique. On pourrait ajouter à ceux-ci beaucoup d'autres aspects : je pense à ce que l'on pourrait appeler désormais «l'anthropologie de l'écriture»", l'étude des écritures communes considérées comme un instrument servant à enquêter sur la mémoire sociale, ou encore à la confluence de l'anthropologie et de la littérature comparée, ou enfin à l'enquête critique menée sur les littératures non occidentales.

Il s'agit, on le voit, d'un domaine d'intérêt infini' ${ }^{4}$. Mais au lieu de continuer à explorer les rapports entre l'anthropologie et la littérature dans l'une de ces perspectives, j'aimerais en proposer une autre. Il ne s'agit pas de mettre en relation les ouvrages d'anthropologie avec les textes littéraires. J'utiliserais plutôt un modèle qui plonge ses racines dans le besoin de raconter, dans la nature de la narration conçue comme condition de l'être au monde de l'homme, et enfin dans ce qu'elle engendre, à savoir les différents genres de narration : la narration religieuse, mathématique, la narration de l'anthropologie et celle de la physique, la narration littéraire et celle de la politique, mais aussi la narration de la fête et celle du quotidien, celle des autres, mais enfin et surtout la narration plus ou moins silencieuse de son propre moi. Chacune est nécessaire à sa manière, chacune, avec ses règles et sa rhétorique, avec une histoire qui les lie aux autres, ou les engendre l'une l'autre.

En somme, il ne s'agit pas ici du rapport entre romans et essais, mais du rapport entre la manière dont la littérature raconte le monde et la manière dont le fait l'anthropologie, entre le besoin pour l'une d'une certaine forme de vérité, et le besoin pour l'autre d'une autre forme de vérité.

Il faudrait formuler à ce propos au moins une prémisse et trois observations. D'abord, le terme "besoin» (celui de la narration littéraire ou de l'anthropologie) ne doit pas être pris dans un sens figuré, tant il indique quelque chose de plus concret et spécifique : pour vivre, nous avons besoin de littérature et de science (y compris l'anthropologie), de même que nous avons besoin de la narration du sens commun ou de celle de la religion',

3. A. Iuso, Q. Antonelli (dir.), Vite di carta, Naples, L'ancora del Mediterraneo, 2000.

4. P. Benson (dir.), Anthropology and Literature, Urbana, University of Illinois Press, 1993; V. Daniel, J. Peck (dir.), Culture/Contexture, Exploration in Anthropology and Literary Studies, Berkeley - Los Angeles - Londres, University of California Press, 1996; R. De Angelis, Between Anthropology and Literature : Interdisciplinary Discourse, Londres, Routledge, 2002; Ph. Dennis, W. Aycock (dir.), Literature and Anthropology, Lubbock, Texas Tech University Press, 1989; A. Krupat, Ethnocriticism : Ethnography, History, Literature, Berkeley, University of California Press, I992; M. Manganaro (dir.), Modernist Anthropology, from Fieldwork to Text, Princeton, Princeton University Press, 1990; F. Poyatos, Literary Anthropology : a New Interdisciplinary Approach to People, Signs and Literature, Amsterdam, J. Benjamins, I988.

5. L. Wolpert, Six Impossibile Things Before Breakfast, Londres-New York, W. W. Norton, 2006; V. Girotto, T. Pievani, G. Vallortigara, Nati per credere, Turin, Codice Edizioni, 2008. 
quelle que soit la vérité du contenu de cette narration. Le rappel aux sciences de l'esprit ne sert pas seulement à jouir de bonnes métaphores, mais à comprendre le dernier arrière-plan de la condition humaine.

Passons maintenant aux observations préliminaires : d'abord, toutes les narrations, de la physique à la religion, des mathématiques à la philosophie, ont été à des moments différents et de différente manière des narrations littéraires, toutes ont imaginé plus qu'elles n'ont démontré. Pour les "domaines du savoir», revendiquer leur propre statut scientifique a souvent signifié prendre ses distances à l'égard de la narration littéraire. Mais c'est vers ce genre de narration que les sciences glissent sans s'en rendre compte, elles y reviennent souvent dans les moments de crise ou de transition, quand elles «imaginent des hypothèses» pour tenter de nouvelles voies. On pourrait écrire l'histoire de toutes les sciences à partir de leur rapport avec l'aura accordée au littéraire.

Ensuite, toute narration est nécessairement narration du monde et de soimême dans le monde, toute narration raconte aussi celui qui raconte, évidemment dans une mesure différente selon qu'il s'agisse de raconter le monde sub specie mathematicae, de la narration des chimistes et des physiciens, celle des psychanalystes, des philosophes ou des religieux. La phrase "Madame Bovary, c'est moi » est toujours valable, et dans tous les domaines. Les différentes narrations sont le moyen de percevoir et d'affronter le paradoxe qui en découle et dont il a été question plus haut. Et puisqu'il est impossible de résoudre ce paradoxe, les narrations sont les différents moyens de le contourner.

Évidemment, nous n'y arrivons pas toujours, ou il n'est pas facile d'y arriver : dans la narration poétique, nous acceptons sic et simpliciter de baigner dans le non-dicible; dans la narration religieuse, nous acceptons de baigner dans le mystère, mais nous déléguons à d'autres la possibilité de raconter; dans la narration du sens commun, nous avons confiance en ce grand narrateur qu'est le "on" impersonnel; dans la narration scientifique, nous essayons de prendre nos distances avec le monde, pour essayer de le voir de l'extérieur, mais contrairement à ce qui se produit dans la narration littéraire, nous devons respecter des règles de fer, des codes, la cohérence. Et puis, il y a précisément la narration littéraire (et plus généralement la narration artistique), la mère de toutes les narrations, le chemin le plus bref pour arriver à la "vérité»: d'une part, l'auteur y est l'artisan des mondes, il les invente, tout en restant à l'extérieur de ceux-ci, comme l'homme de science; de l'autre, il pense avec la tête de ses personnages, il est à l'intérieur du monde que constitue son œuvre.

Enfin, si nous ne voulons pas faire de poésie ou raconter le monde d'un point de vue religieux (des narrations proches sur bien des points), le système 
le plus fréquent consiste à se placer résolument à l'extérieur, à feindre de regarder le monde de l'extérieur, à oublier notre participation au monde dont nous parlons. Pour les sciences dures, ce type de regard, «le regard de l'extérieur» implique la garantie de l'objectivité scientifique. Pour nos disciplines, c'est plus difficile : nous devons rester hors du monde que nous racontons, mais nous devons aussi démontrer que nous l'avons traversé, que nous y avons été, que nous avons observé les idées qui sont dans la tête des autres, comme les chimistes observent la combinaison des éléments. Car nous ne pouvons démontrer que ce monde existe que si nous y avons été.

Etre à la fois à l'extérieur et à l'intérieur du monde dont nous parlons, quelle ambition! Pour la satisfaire, la littérature a beaucoup à nous apprendre. Mais il faut faire particulièrement attention à ne pas confondre les narrations des «sciences humaines» avec celles de la littérature, et, en particulier, les narrations littéraires avec celles de l'anthropologie. Elles ont beaucoup de choses en commun : chacune d'elles raconte des mondes autres, en quelque sorte chacune d'elles voyage, s'éloigne de son propre monde et y revient pour raconter sa propre expérience. Mais il est permis aux romanciers d'inventer des histoires, d'imaginer des mondes, alors que pour autant qu'on veuille souligner leur caractère interprétatif, nos disciplines doivent tendre à expliquer. Du moins, depuis qu'il est devenu chercheur de terrain, depuis qu'il a pris pour base l'ethnographie, l'anthropologue doit revenir raconter ce qu'il a vu "de ses propres yeux» et lui donner un sens. La littérature raconte des mondes possibles, ou, dans un sens plus large, l'existant; l'anthropologie tend à la réalité, à raconter l'existant réalisé et, comme les autres sciences, à l'expliquer. L'homme a besoin de ces deux vérités : celle de l'imagination, mais aussi celle de la science (qui constituent, chacune, un aspect de l'intentionnalité dont nous avons parlé plus haut). Il n'est pas permis à l'anthropologie de s'en tirer en disant, comme Don Quichotte, qu'en ce monde il arrive des choses qui ont plus l'air d'être vraies que d'avoir un sens.

Les romanciers jouissent d'une liberté qui est refusée aux anthropologues. $\mathrm{Si}$ j'avais écrit (il y a presque trente ans) que dans les villages de San Vincente du cap Vert, il a plu comme jamais (se Deus quiser!), j'aurais dit le faux tout simplement, alors qu'un romancier, dans ce cas Germano Almeida, peut faire de la même affirmation le début d'un brillant récit anthropologique ( $O$ testamento do Sr. Napomuceno da Silva Araújo ${ }^{\circ}$ ). Certes, si l'on considère (avec Wittgenstein) en quoi et combien l'imagination constitue un instrument indispensable pour comprendre la manière dont les autres voient le monde,

6. G. Almeida, O testamento do Sr. Napumoceno da Silva Araújo, Lisbonne, Caminho, I99I. 
et, par ailleurs, en quoi l'imagination (des romanciers) est toujours liée à la réalité, les choses se compliquent. J'admets sans difficulté que l'on comprend sans doute mieux ce qu'il en était du cap Vert en lisant un roman d'Almeida, qu'en lisant mes travaux.

Tout au long de son histoire et en plusieurs occasions, l'anthropologie s'est interrogée sur sa propre difficulté de raconter. Vincent Crapanzano appelle cela le "dilemme d'Hermès" : il faut recommencer à raconter, dans un monde que nous avons cessé d'habiter pendant un certain temps, ce que nous croyons avoir compris (de ce que d'autres que nous ont pensé de leur propre monde). Et encore, à la suite de Wittgenstein, nous pourrions ajouter qu'une recherche ne peut être considérée comme telle qu'à partir du moment où elle nous a changés, où nous nous sommes approprié une autre manière de voir le monde. Ceci est valable, à mon avis, aussi bien pour une recherche en littérature ou en histoire, que pour une recherche en anthropologie. Selon moi, quiconque passe plusieurs années à étudier Pirandello en sort nécessairement un peu "pirandellisé». Et je peux assurer que quand on vit un an dans une île de l'Atlantique, on revient de ce séjour différent de ce qu'on était auparavant (même si l'on se réadapte à l'état antérieur).

Rien de nouveau. Dans un volume récent, Il cristallo e la fiamma ${ }^{7}$, j'ai essayé de repenser les différentes manières qu'ont adoptées les anthropologues pour se situer par rapport au paradoxe de la connaissance et de la narration de l'autre et les différents résultats de la narration anthropologique. On pourrait envisager un travail analogue sur toute autre discipline et, dans certains cas, cela a déjà été fait ${ }^{8}$. On a étudié par exemple la manière dont les romanciers (ou les auteurs d'épopées, voire les poètes) ont convaincu le lecteur à entrer dans l'univers de leur propre narration, ou encore, on a étudié les différentes formes stratégiques de la narration historique?.

C'est sur ce type d'observations que se fonde l'apport théorique de Clifford Geertz ${ }^{\text {Io }}$. Il a eu le mérite d'avoir placé au premier plan le "nœud" du récit anthropologique; sa limite - surtout dans Works and Lives - est de penser que ce nœud puisse être dissous grâce à des stratégies, à des stratagèmes rhétoriques de type littéraire qui permettent d'entrer et de sortir du monde que l'on raconte.

Se situer entre l'expérience vécue (to be there) et racontée (to be here) est plus difficile à vivre que ne le pense Clifford Geertz. Ce n'est pas une question

7. A. Sobrero, Il cristallo e la fiamma, Rome, Carocci, 2009.

8. Voir T. Mitchell, On Narrative, Chicago-Londres, University of Chicago Press, 198I.

9. À partir de H. White, Metahistory: The Historical Imagination in Nineteenth-Century Europe, Baltimore-Londres, Johns Hopkins University Press, 1973.

Iо. Cl. Geertz, Works and Lives, Stanford, Stanford University Press, 1988. 
que l'on peut résoudre sur le plan pragmatique et rhétorique. Au contraire, le souci de l'homme de sciences anthropologue, voire, d'une certaine façon, son plus grand souci, est depuis toujours de ne pas être confondu avec un romancier. "Ceci n'est pas un roman...", écrivaient et continuent d'écrire les anthropologues au début de leurs comptes rendus. Mais qui peut s'en porter garant? Quel est le critère de vérité? Les sciences dures doivent respecter les contraintes et les contrôles, elles sont obligées de répondre à une cohérence de discipline, mais elles peuvent compter, dans la plus grande partie de leur histoire, sur l'évidence de la preuve et sur la cohérence des démonstrations. Mais dans le cas de nos disciplines? Notre discipline jumelle - l'histoire - a ses documents, mais qui pouvait contrôler en 1922 que ce que Malinowski disait sur les échanges étranges des Trobriandais répondait à vérité? Qui aurait pu garantir que son ethnographie n'était pas du roman? On peut objecter qu'il ne reste désormais plus beaucoup d'îles lointaines ni beaucoup d'exotisme. Mais quel est le critère de vérité qui permet aujourd'hui aux anthropologues de raconter la vie des banlieues aux habitants des quartiers du centre-ville, ou aux adultes ce qui passe par la tête des jeunes?

Pour penser "différemment", nous avons besoin de l'imagination des romanciers. Mais nous devons aussi prendre nos distances par rapport à leur art et prouver que nous disons le vrai et que ce vrai peut être attesté par notre présence, au milieu de ces hommes et surtout «dans leur tête». En somme, la narration (l'imagination) littéraire est à la fois notre meilleure alliée et notre pire ennemie.

Malinowski est généralement reconnu comme le père de l'ethnographie scientifique, mais on ajoute toujours qu'il y a un revers de la médaille : ce sont ses journaux intimes ${ }^{\text {II }}$. Cette manière de remâcher sa propre histoire, son vécu : l'envie d'écrire "un roman de soi», la bataille contre le vice absurde de lire des romans. Malinowski était obsédé par l’idée que son travail écrit pût être confondu avec un roman. Il considérait son amour pour les romans comme un vice à mettre sur le même plan que la drogue, l'alcool, la sexualité sans frein. Dans les notes de Mailu, datées du 2I octobre I9I4, il nous dit qu'il avait fini Vanity fair et lu Romance sans pouvoir bouger, comme drogué. Ou encore, à la date du 27 septembre 19I4, qu'il était sous l'influence de Tunnel qu'il avait lu pendant des heures jusqu'à la fin; il se repromettait de ne pas lire de romans. Et en effet il maintint sa promesse pendant quelques jours. Puis il retomba dans l'erreur ${ }^{12}$.

II. B. Malinowski, A Diary in the Strict Sense of the Terme, Londres, Routledge \& Kegan, 1967.

I2. Ibid. 
Par amour ou par force (par la force des choses, dans son cas), il avait été là-bas trois années durant et malgré son amour pour les romans et les romanciers, il ne voulait pas être confondu avec Balzac, Stevenson ou Conrad pour lesquels il éprouvait une sorte de haine/amour; « je veux être le Conrad de l'anthropologie» : il remporta ce combat difficile, non sans pertes considérables.

Malinowski peut être considéré à juste titre comme notre père à tous, précisément parce qu'il a réussi à cacher ses journaux intimes dans un tiroir, à faire un saut qualitatif (n'était-ce pas ce que nous enseignait la logique de Russell ?) et à construire (à la suite de trébuchements significatifs) ce style ethnographique sec, essentiel, cette "écriture de l'extérieur" que demandait et demande encore aujourd'hui l'académie. Pour parler brutalement, il a réussi à rendre scientifique notre discipline à travers cet étrange mécanisme autoréférentiel selon lequel nous ne répondons qu'aux questions que nous savons poser. Une telle position a facilité, d'une certaine manière, le travail des anthropologues que Clifford Geertz considère dans Works and Lives: Ruth Benedict, Lévi-Strauss, Evans-Pritchard. Ce sont tous de grands fondateurs de discours, mais de discours répondant au langage de leurs propres lecteurs. Ils ont une manière de raconter from our point of view. La bataille des anthropologues contre la littérature a été gagnée, en partie du moins.

Quelques années plus tard, Michel Leiris mène le même combat, ou du moins un combat analogue qui se termine par une défaite. Il fut plus difficile, car contrairement à Malinowski, Leiris a voulu le mener sur les deux fronts : contre ceux qui voulaient mettre en doute sa vérité et confondre son travail avec un roman, mais aussi contre la logique de la science dont nous venons de parler.

Comme chacun sait, la production littéraire de Leiris est une autobiographie, un récit de vie en plusieurs volumes (Philippe Lejeune, Jean Jamin et Catherine Maubon l'ont parfaitement analysér $\left.{ }^{\mathrm{r}}\right)$, à partir d'Aurora, en passant par L'Âge d'homme jusqu'à Frêle Bruit, le dernier des quatre volets de sa longue autobiographie, La Règle du jeu: une étrange autobiographie, non linéaire, qui revient toujours sur elle-même, en glissant (terme cher à Leiris) sur le son des mots. Lordre est à lire dans la forme même de l'écriture, les mots se répondent, ne deviennent des idées que l'espace d'un instant pour redevenir bien vite des sons, tout au plus des images, comme si les idées se cachaient derrière des sons, comme si on écrivait pour ne pas penser, voire pour masquer l'échec de sa propre vie aux yeux des autres. Jean Jamin

I3. J. Jamin, Lire Leiris. Autobiographie et langage, Paris, Klincksieck, 1975; C. Maubon, introduction à Michel Leiris scrittore-etnografo, dans C. Maubon (dir.), Michel Leiris, L'occhio dell'etnografia, Turin, Bollati Boringhieri, 2005. 
a magistralement reconstruit le mouvement de l'écriture de Leiris, ce voile qu'il étend sur sa propre existence, alors même qu'il la raconte de manière obsessionnelle ${ }^{\mathrm{I}}$.

L'Afrique fantôme n'est alors qu'une exception apparente. Dans les manuels d'anthropologie, on ne cite généralement Leiris que comme l'auteur de cet ouvrage, auquel on ajoute quelque chose comme "compte rendu, journal de l'expédition menée par Marcel Griaule de Dakar à Djibouti de I93I à I933". C'est en effet un livre difficile à lire et à classer : six cents, sept cents pages heurtées, selon le rythme d'un journal intime écrit au jour le jour, une suite de petites observations sur l'état d'âme, la santé physique de l'auteur; des images sexuelles, des rêves, des pensées éparses, des réflexions sur les sentiments éprouvés pour les indigènes. Autrement dit, peu d'observations sur ce que Griaule aurait considéré comme étant digne de notes ethnographiques.

Dans le journal intime de ces deux années, Leiris essaie de mener l'opération diamétralement opposée à celle d'Aurora et de ses journaux, dans lesquels il tente de défier le temps et de tromper son moi fragile en niant sa mutation, en en retrouvant la continuité dans les moindres signes. Dans l'expérience africaine, au contraire, tout moment doit être non pas un temps retrouvé, mais un temps entièrement neuf et, donc, un non-temps, un temps virtuel, éternel. Il s'agit d'une victoire sur la maladie du temps, rendue possible par un voyage sans haltes, par un cheminement qui ne connaît ni passé ni futur, qui n'accorde pas de temps au temps.

Au sortir de ces limbes, conseillé par mon médecin et pensant moi-même qu'il me manquait d'avoir un peu vécu à la dure, je saisis l'occasion de faire un long voyage et partis pour près de deux ans en Afrique, comme membre d'une mission ethnographique ${ }^{15}$.

Le récit est chronologique et l'on comprend qu'il doit l'être totalement : de manière obsessionnelle, il est écrit pendant vingt mois, jour après jour, sans en sauter un seul, et très souvent, vers la fin, quand la vie multiplie les propres questionnements, d'heure en heure. Car on ne sait pas, ou on ne peut pas, avoir le temps de répondre.

Le voyage dure du I9 mai I93I au 4 février 1933. Comme je l'ai déjà écrit ${ }^{16}$, le journal peut se diviser en six parties dont je peux donner une idée de la manière suivante : sautes d'humeur, alternance d'états de frustration et d'exaltation du départ, aussitôt suivie de la frustration pour un voyage qui n'apparaît pas si différent de celui d'une troupe de cirque; puis approche du territoire des Dogons, sur lesquels à Paris on fantasmait tellement; le voyage

I4. J. Jamin, ouvr. cité.

15. M. Leiris, L’Âge d'homme, Paris, Gallimard, 1939, p. 199.

I6. A. Sobrero, ouvr. cité. 
qui recommence, une sensation non pas d'arrivée mais de nouveau départ, et moins de quinze jours après, de nouveau la sensation d'inutilité, d'être l'acteur d'une violence continuelle; les mots avec Griaule et d'autres membres du groupe. Peut-être le point le plus bas de tout le voyage. Et encore une succession de sentiments contradictoires, jusqu'à Gondar, jusqu'à l'exaltation, à la sensation et la peur de vivre sa propre vie pour la première fois :

Après des mois de chasteté et de sevrage sentimental, séjournant à Gondar, je fus amoureux d'une Éthiopienne [...] Très belle de visage mais la poitrine ravagée, elle était engoncée dans une toge d'un blanc généralement plus que douteux, sentait le lait suri $[\ldots]^{17}$

À Gondar, l'écriture de Leiris devient alors plus dense, pour éviter de se laisser emporter dans quelque fausse profondeur. Mais c'est justement à la fin de la première partie, au printemps 1932, que Leiris expose la préface potentielle (préface et pas démarche) pour une recherche qui n'aura jamais lieu. On ne peut témoigner de la vérité du monde autre si on ne témoigne pas de sa propre existence là-bas, en son sein. Et l'on ne peut témoigner la vérité de sentiments autres, d'une autre manière d'être au monde, si on ne témoigne pas de sa propre relation intime avec ces sentiments. Selon Leiris, c'est à travers le maximum de subjectivité que l'on atteint l'objectivité; en exposant à la lumière du jour le coefficient personnel, le calcul de l'erreur, que l'on peut le mieux garantir l'objectivité.

Je ne pense pas que Leiris ait été le premier représentant d'une ethnographie critique à l'égard de la forme classique du savoir anthropologique, ni qu'il ait été le premier à se rendre compte de la difficulté de raconter un monde différent du nôtre. Ce qui le distingue néanmoins, c'est qu'il n’a pas cherché de raccourcis ni de solutions. Il s'agit pour lui d'assumer pleinement cette difficulté mais, selon son style, de ne pas lui chercher de solution, d'en explorer plutôt la nature en la portant à ses conséquences extrêmes.

Leiris est magistral dans cette "écriture de l'intérieur", écriture qui ne se pose jamais au-dehors du monde dont il parle et qui, à chaque pas, réaffirme que la certitude de l'existence de ce monde passe seulement par l'extrême subjectivité de l'expérience. Il l'avait déjà écrit quelque temps auparavant dans Aurora:

Il m'est toujours plus pénible qu'à quiconque de m'exprimer autrement que par le pronom Je; non qu'il faille voir là quelque signe particulier de mon orgueil, mais parce que ce mot Je résume pour moi la structure du monde. Ce n'est qu'en fonction de moi-même et parce que je daigne accorder quelque attention à leur existence que les choses sont ${ }^{18}$.

I7. M. Leiris, L’Âge d'homme, ouvr. cité, p. 199-200.

I8. M. Leiris, Aurora, Paris, Gallimard, I946, p. 39. 
Jean Jamin a étudié le style de cette "écriture de l'intérieur» dans les romans autobiographiques. Ne pouvant m'y arrêter, je rappelle brièvement que c'est une technique fondamentale du surréalisme : il faut d'abord sentir les mots, leur son, leur goût, l'aura qui les entoure, il faut les percevoir comme étant doués d'une vie propre et sentir qu'on fait partie intégrante de cette vie, afin que de ces mêmes mots puissent naître des sensations, des perceptions, des souvenirs soudains, inattendus. C'est ainsi que les mots deviennent "nôtres» et nous sommes nous-mêmes parce que nous sommes au cœur de cette écriture.

En disséquant les mots que nous aimons, sans nous soucier de suivre ni l'étymologie, ni la signification admise, nous découvrons leurs vertus les plus cachées et les ramifications secrètes qui se propagent à travers tout le langage, canalisées par les associations de sons, de formes et d'idées. Alors le langage se transforme en oracle et nous avons là (si ténu qu'il soit) un fil pour nous guider, dans la Babel de notre esprit ${ }^{19}$.

Dans L'Afrique fantôme, la technique de "l'écriture du dedans» utilise des instruments communs à beaucoup de surréalistes (Bataille, Queneau surtout), mais elle en ajoute de nouveaux, ne serait-ce que parce que le monde dont il est question est plus éloigné et plus étranger à l'auteur et au lecteur. Reste le caractère continu et obsessionnel des temps d'écriture, jour après jour. Néanmoins, ce qui frappe le plus, c'est qu'il n'y a aucun rappel des jours passés. Rien non plus quant aux jours à venir. Le journal intime est «journal» dans le sens le plus strict du terme, un présent continu, qui se dilate pendant presque six cents jours, comme si le temps ne passait jamais, comme si la vie était réellement suspendue, repliée sur elle-même. De même, les références à la vie européenne, aux événements et aux personnes sont rares, aussi rares que les références au paysage, presque inexistant. Comme dans Au cour des ténèbres, l'Afrique n'y apparaît presque pas. Le véritable objet du journal semble être le journal lui-même :

5 avril [1932] : [...] C'est presque comme si j’avais eu l'idée du voyage exprès pour le rédiger [...] Plus assommant qu'un journal sont deux journaux. Inutile d'ajouter à l'ennui de celui-ci l'ennui d'un «journal du journal $»^{20}$.

Le paysage est absent, ou plutôt, le paysage n'est présent qu'à travers des champs étroits, des scènes coupées, interrompues, comme il arrive justement à celui qui observe de l'intérieur et ne voit pas l'ensemble de la scène, mais déplace son regard rapidement, passe rapidement d'un épisode à un autre. La technique est bien connue en littérature (en fait foi le célèbre dixième épisode

19. M. Leiris, Brisées, Paris, Mercure de France, 1966, p. II.

20. M. Leiris, L’Afrique fantôme (désormais AF), Paris, Gallimard, coll. «Tel», 1988, p. 268. 
d'Ulysse). L'espace du paysage ne peut coïncider avec l'espace de l'écriture. Leiris en est conscient :

I8 mars [1932] : [...] Depuis hier, discussions sur les carnets de route de Gide [...]. Je les défends pour le principe [...]. Mais toutes les descriptions, si brèves soient-elles, sont décidément bien vaines. On ne peut retracer un paysage, mais tout au plus le recréer; à condition, alors, de n'essayer aucunement de décrire. [...] Écrire un livre de voyage n'est-il pas, il est vrai, une absurde gageure, par quelque but qu'on s'y prenne? (AF, p. 248).

L'espace du regard de l'intérieur ne peut être qu'un espace de détails. Nous n'avons jamais de paysage ni, donc, de grandes scènes. À Gondar, quand Leiris est obligé de rendre compte des fêtes du village, l'écriture devient haletante : c'est une course engagée contre le temps. Comme il le dira en d'autres occasions, "[l]a faute en est à la lenteur de cette plume qui peine toujours comme pour remuer des montagnes, quand je la voudrais si cursive ${ }^{21} »$.

Enfin, il y avait ce regard continuellement tourné vers soi, effrontément, comme une provocation :

Ces notes [écrit-il dans l'introduction de l'édition de 1934] «ne sont pas un historique de cette mission. Simplement journal intime. On trouvera qu'en maints endroits je me montre particulier, chagrin, difficile, partial - voire injuste - inhumain (ou «humain, trop humain"), ingrat, faux frère, que sais-je? Mon ambition aura été, au jour le jour, de décrire ce voyage tel que je l'ai vu, moi-même tel que je suis ${ }^{22}$.

Tout semble préparer les pages finales, les pages sur la rencontre avec Emawayish à Gondar : belle, "[...] encore qu'à son allure de princesse se mêle un certain côté succube, à chair molle, moite, froide, qui m’écœure, en même temps qu'il me fait un peu peur $[\ldots]$ » $(A F$, p. 430).

Toute distance ethnographique et tout reste de préoccupation à l'égard de Griaule, mais aussi de son propre rôle, sont laissés de côté. Il faut admettre qu'indépendamment des rapports personnels, du jugement sur le colonialisme et sur le rapport avec les indigènes, des engagements différents de chacun par rapport aux objectifs de l'expédition, Marcel Griaule avait d'excellentes raisons de considérer les pages de Leiris comme étrangères à toute perspective ethnographique. Pensons à ce qu'écrivait dans ces années-là Marcel Mauss dans son Manuel d'ethnographie (1926). Selon lui, il n'y a pas de place pour l'intuition dans la science ethnologique, science fondée sur la constatation et sur la statistique.

La description, comme toujours, avance par scènes interrompues, par détails, avant de devenir extrêmement dense; les scènes sont plus confuses,

21. M. Leiris, Biffures Paris, Gallimard, 1988, p. 24I.

22. M. Leiris, L'Afrique fantôme, Paris, Gallimard, coll. «Les Documents bleus», I934, page non foliotée. 
pleines de voix, de couleurs, d'odeurs. L'odeur des animaux tués pour le sacrifice et dont on boit le sang; les intestins autour de la tête; les formules magiques répétées jusqu’à la transe :

27 août [1932] : J'ai besoin de tremper dans leur drame, de toucher leur façon d'être, de baigner dans la chair vive. Au diable l'ethnographie! le carnet d'Abba Jérôme - sur lequel je lui fais noter au vol ce que dit la vieille, ou bien sa fille, ou bien quelqu'un de l'entourage - m'est un monde de révélations dont la traduction, chaque fois, me plonge dans le délire... (AF, p. 436)

Quelques années plus tard, Lévi-Strauss écrira son journal brésilien. LéviStrauss est à mon avis tout aussi incompréhensible si l'on ignore sa formation en milieu surréaliste, mais dans ce grand chef-d'œuvre qu'est, pour d'autres raisons, Tristes tropiques, on ne trouve pas d'odeur, de même qu'on ne trouve pas de sentiment qui ne soit passé au crible de nos sens.

"L'écriture de l'intérieur " atteint son apogée dans les dernières pages. Leiris se situe dans la marge extrême de ce monde : «Nous sommes des affiliés!» ditil, mais il reste dans la marge : ce n'est pas le monde parisien de Madame Edwarda (Bataille) ni les femmes d'Aurora, c'est un monde dont le séparent «des questions de peau, de civilisation, de langue». Elles sont les premières à «jouer» un rôle : elles l'ont tenu au seuil de leur vérité. Pourtant, elles lui ont fait entendre que cette vérité existe pour elles : «I4 septembre [1932] : [...] Et jamais je n'avais senti à quel point je suis religieux; mais d'une religion où il est nécessaire qu'on me fasse voir le dieu...» ( $A F, \mathrm{p} .463)$.

Faire un pas de plus est impossible. Mais ce pas a suffi à le séparer définitivement du monde de Griaule et de la possibilité de revenir raconter.

Malinowski n'écrira jamais de "roman du propre moi» auquel il avait pensé se consacrer à son retour en Europe; il écrira des essais devenus célèbres, sur lesquels se fonde l'écriture de l'ethnographie, la base de la nature scientifique de l'anthropologie occidentale. Leiris n'écrira jamais un roman, sinon le long roman de son «moi»; mais il n'écrira pas non plus d'ouvrage d'anthropologie. Malgré son voyage en Afrique et la position qu'il occupera au musée de l'Homme, il restera enfermé dans ses propres sortilèges. L'essai de I958 intitulé La possession et ses aspects théâtraux chez les Éthiopiens de Gondar reprendra la dernière partie de L'Afrique fantôme, en sacrifiant les pages sur Emawayish aux critères des commandes académiques éventuelles.

Toute possibilité de rapport avec le monde extérieur est interrompue :

${ }^{\mathrm{er}}$ octobre [1932] : [...] Je ne parle pas. Avec qui parlerais-je? Je mange des grains qu'on me donne, bois le café qu'on me tend. Je regarde ces trois choses : le carnet d'Abba Jérôme, le diaphragme du mouton, le genou nu de Emawayish, et je sens plus que jamais mon irrémédiable isolement. C'est comme si ces trois points, formant un triangle dans ma tête (du fait que je suis seul à connaitre tous leurs 
liens), coupaient autour de moi l'univers au couteau comme pour m'en séparer et m'enfermer à jamais dans le cercle - incompréhensible ou absurde pour quiconque - de mes propres enchantements... (AF, p. 504)

Des jours de désespoir s'ensuivirent. Temps inutile. Puis finalement le retour : I6 février [1933] : [...] Le bateau oscille légèrement. J'ai l'esprit net, la poitrine calme. Il ne me reste rien à faire, sinon clore ce carnet, éteindre la lumière, m'allonger, dormir, - et faire des rêves... (AF, p. 648) 